\title{
Políticas públicas en Educación y Comunicación: Debates y avances
}

\section{Education and Communication Public Policy: Discussions and Developments}

\author{
Ana Isabel Rodríguez-Vázquez \\ Universidad de Santiago de Compostela (España) \\ Rosa García-Ruiz \\ Universidad de Cantabria/Universidad Nebrija (España) \\ Ignacio Aguaded \\ Universidad de Huelva (España)
}

Las relaciones de la ciudadanía con los medios de comunicación son cada vez más complejas y hacen necesario planificar y proyectar la educación en un contexto hipermediático, planteando nuevos retos: en la educación se integra la responsabilidad de formar a los ciudadanos para consumir y producir en un ecosistema de medios con capacidades y potencialidades para los nuevos públicos. En esta relación estratégica entre educación y comunicación, las políticas públicas deben fortalecer las condiciones que favorezcan la capacitación personal, uniendo la creatividad con la comprensión de las estructuras, procesos y técnicas que refuercen un consumo crítico. Se ofrece una panorámica de los avances que se han producido en torno a la alfabetización en los nuevos medios, a partir de un análisis de contenido desde la definición de este concepto por la UNESCO en 1979 has-
Relations between citizens and mass media are becoming increasingly complex, making it necessary to plan and design education in a hypermediatic society, where new challenges arise: education has got the primary responsibility of training citizens to consume and produce in a media ecosystem with great capacities and potentialities for new audiences. In this strategic relation between education and communication, public policies should strengthen a framework that encourages personal empowerment, combining creativity with understanding of the structures, processes and techniques that enhance critical consumption. This paper offers an overview of the advances that have occurred with regard to the new media literacy, based on content analysis, from the definition of this concept by UNESCO in 1979 to the present, through "New Curriculum for Teachers 
ta la actualidad, pasando por el New Curriculum for Teachers on Media and Information Literacy de 2011 y el Mapping Media Education in the World de 2009. También se recogen los principales debates abiertos en un contexto global que debe reforzar el valor de la diversidad ante el gran desafío de la convergencia. El empoderamiento de la ciudadanía es un valor indudable que favorecerá su inclusión cultural y su participación crítica en la esfera mediática convirtiéndose en prosumidores mediáticos y digitalizados, para lo que las políticas públicas en educación y comunicación deben seguir avanzando y sumando esfuerzos.

Palabras clave: educación en medios, políticas públicas, convergencia, análisis de contenido, alfabetización mediática. on Media and Information Literacy" of 2011 and "Mapping Media Education in the World" of 2009. Additionally, we deal with the main discussions held in a global context because it is imperative to reinforce the value of diversity in the face of the great convergence challenge. The empowerment of citizenship is an unquestionable value that will be beneficial to their cultural inclusion and their critical participation in the media sphere, so that they become media and digital prosumers, and to this effect public policies in education and communication must continue advancing and joining efforts.

Key words: media education, public policy, convergence, content analysis, media literacy.

$\mathrm{L}$ as tecnologías de comunicación han supuesto la irrupción de la digitalización en el universo de los escolares: desde la televisión los jóvenes se desplazan con fuerza hacia el ordenador, las tabletas o los teléfonos móviles, los cuales canalizan más del 90\% de la circulación de los contenidos mediáticos que apenas se consumen ya en otros soportes más tradicionales como el papel o los simples receptores de radio o TV.

Como reconocen numerosos estudios sobre hábitos de consumo, los jóvenes pasan más tiempo en los medios de comunicación digitales que en cualquier otra actividad (Gutiérrez y Gómez, 2015), lo cual no quiere decir que su uso sea correcto, tal y como matizan Martens y Hobbs (2015). Es el paradigma de la convergencia: fusión del tiempo y el espacio de ocio y de estudio, del uso personal y aplicado al aprendizaje que se hace de los recursos comunicativos, de la utilización de múltiples soportes incluso simultáneamente (consumo multitasking), lo que obliga a la educación mediática actual a transformar y convertir este reto en una oportunidad.

Por eso, aprovechar la gran fuerza de unir comunicación y educación en el desarrollo individual y social sitúa este tema en el epicentro del interés científico en los entornos de ambas disciplinas. Proyectos interdisciplinares y transdisciplinares reúnen la dimensión teórico-práctica de las variadas formas de enseñar 
y aprender en un entorno mediatizado por la fuerza de la comunicación en los procesos de socialización. Propuestas como el ecosistema de formación de Álvarez-Arregui y Rodríguez-Martín (2015) y Álvarez-Arregui et al. (2017) resultan especialmente interesantes para favorecer la convergencia y la implementación en los estudios universitarios, lo que contribuye al desarrollo de la alfabetización mediática. Los educadores y los alumnos son alternativamente emisores y receptores en un proceso de aprendizaje continuo y compartido que tiene como principal objetivo desarrollar la capacidad de demostrar un pensamiento crítico. Es el valor añadido de la alfabetización mediática (media literacy), que, con la explosión tecnológica de la digitalización, acelera el proceso conversor de un receptor pasivo en un emisor permanentemente conectado y posiblemente activo. Así lo demuestra la experiencia llevada a cabo con escolares adolescentes chilenos, en la que se potencia la producción mediática digital y el aprendizaje como prácticas culturales, lo cual favorece el desarrollo de la producción mediática creativa desde una perspectiva pedagógica, que traspasa el contexto escolar (Valdivia-Barrios, Herrera y Guerrero, 2015); o la propuesta de Hobs et al. (2013), en la que se evidencia cómo esta convergencia educomunicativa implementada en prácticas pedagógicas, que facilitan el consumo y producción de mensajes audiovisuales, favorece en los jóvenes también su compromiso ético y cívico como ciudadanos.

En esta última década (desde 2010) se pueden localizar en el mundo de la comunicación dos situaciones que son la clave del desarrollo que se produjo desde entonces en la oferta y el consumo mediáticos, especialmente aquel que afecta más a los jóvenes: por un lado, la digitalización que muchos países afrontan de sus emisiones televisivas (switch off —apagón analógico-, encendido digital y consolidación de las plataformas Over-The-Top/OTT); y, por otro lado, la explosión del fenómeno de las redes sociales como soporte del avance de la conectividad. Se abre, entonces, el nuevo escenario de la convergencia en el que se han de revisar las políticas públicas y también el rol que debe cumplir la educación en medios tanto en las aulas como fuera de ellas. En el primer caso, desde 2012 y por un período de dos años, se realizó el proyecto Emedus (European Media Literacy Education Study), en el que se analizaba comparativamente la educación en medios de los currículos nacionales de los 27 países de la UE.

Los resultados de este trabajo permiten tener una referencia geográfica de contraste con otra de las grandes regiones mundiales que han trabajado en este tema bajo el amparo de la UNESCO, especialmente desde los años noventa del siglo XX, como Latinoamérica. Y, por supuesto, con las experiencias registradas en Estados Unidos (Tyner, 2013) y en el resto del mundo. Algunas de ellas fueron recogidas en monográficos como "A través de las culturas: una mirada global de la educación mediática" en la revista norteamericana The Journal of Media Litera$c y$, con aportes relevantes respecto a la situación de la alfabetización mediática en diferentes contextos nacionales como el australiano (Dezuanni, 2016), el chino (Lee, 2016), el estadounidense (Goble, 2016) o el canadiense (Andersen, 2016), y en otros países iberoamericanos como Chile (Castro, Renés, y Phillippi, 2016), Brasil, España y Portugal (García-Ruiz, Matos y Borges, 2016). Aquí se ponen de manifiesto muchos de los retos conseguidos en cuanto al desarrollo de expe- 
riencias integradas en los currículos oficiales y otras muchas de la educación no formal, así como otros muchos por resolver.

En este contexto, ¿las políticas públicas avanzan en la dirección correcta? Y, aun sabiendo que la educación en medios traspasa las aulas, ¿qué valor se le da a esta disciplina en los currículos académicos? Si no hay palabra verdadera que no sea unión inquebrantable entre acción y reflexión, como sostenía Paulo Freire (1967), proponer las líneas estratégicas que deben centrar la agenda de los debates a corto y medio plazo sobre comunicación y educación obliga a revisar los avances que se han producido en los cuarenta años de gestión de políticas públicas a escala mundial.

El equilibrio entre reflexión y acción constituye, pues, la línea central de este trabajo que tiene dos objetivos: por un lado, ofrecer los resultados de una investigación que parte del análisis de los principales documentos que marcaron la agenda de las políticas públicas sobre educomunicación, desde 1979 hasta 2019; y, por otro, dejar abiertas algunas líneas para el debate que requerirán la mirada de todos los agentes implicados (stakeholders) en el diseño de las nuevas políticas públicas que den respuesta permanente a los desafíos que se presenten. Se trata de observar cómo las políticas públicas reconocen y se adaptan al nuevo escenario de convergencia, en donde la posibilidad de conexión a internet en múltiples dispositivos, a través de los que se pueden recibir infinidad de contenidos, abre nuevos debates sobre lo que se conoce como "aprendizaje ubicuo, invisible, y entornos personales de aprendizaje donde converge lo aprendido en los libros de texto con lo visto en la televisión, lo escuchado en clase con lo consultado en internet" (Gutiérrez-Martín, 2013: 22).

\section{METODOLOGÍA}

Siguiendo el proceso crítico-reflexivo que propone la propia educación en comunicación, la base metodológica que se aplica para lograr estos resultados se centra en la revisión crítica de los principales documentos públicos que han servido como marco de acción de las políticas de educación y comunicación en medios en las tres últimas décadas, desde la definición de este concepto por la UNESCO en 1979 hasta la actualidad, pasando por el New Curriculum for Teachers on Media and Information Literacy, de 2011, o el Mapping Media Education in the World, de 2009.

El análisis documental ha sido el método seguido para dar respuesta a los objetivos $\mathrm{y}$, concretamente, el análisis de contenido permitió asumir una perspectiva integradora de los documentos analizados, a partir de la cual se definieron dos dimensiones que respondían a los dos objetivos planteados en la investigación, a partir de las que se definieron tres categorías de análisis que se fueron nutriendo de otras categorías emergentes derivadas de la organización de la información obtenida y que permitieron su interpretación y la delimitación de los debates y avances identificados, como el papel de los docentes, la capacitación de los estudiantes, el papel de la educación mediática en el currículum, o la transliteracy en el currículum. 


\section{CUARENTA AÑOS DE POLÍTICAS DE EDUCACIÓN EN MEDIOS}

Desde una óptica transversal, en este campo de intervención cultural y social en permanente construcción, cabe recordar que el inicio de la educomunicación se sitúa en Iberoamérica, donde se localizan los movimientos herederos de las teorías de Paulo Freire alrededor de la educación popular y los estudios de autores como Luis Ramiro Beltrán, Mario Kaplún o Daniel Prieto, entre otros. También es continuadora de los movimientos internacionales sobre educación en medios que han logrado posicionar la educación en comunicación como un valor transversal reconocible en determinadas políticas públicas. Han transcurrido cuarenta años desde que la UNESCO definió la educomunicación como un espacio teórico-práctico que surge por las interrelaciones entre dos campos muchas veces separados: el de la educación y la comunicación, con el fin de poner el potencial de esta unión al servicio del desarrollo social y personal del individuo. Evidentemente se han alcanzado grandes logros para educar en medios a la ciudadanía, sin embargo, quedan aún nuevos retos por superar, que trataremos de categorizar en este trabajo.

Desde hace casi una década se habla de la nueva convergencia ante el protagonismo claro del mundo digital que requiere un nuevo proceso alfabetizador. Como señala Pérez-Tornero (2015), está surgiendo un nuevo paradigma conceptual que plantea un escenario común para la educación en medios tradicional y la nueva interpretación que exige el mundo digital, esto es, la Alfabetización Mediática e Informacional (AMI), que genera a su vez nuevos paradigmas con implicaciones en la investigación y la experimentación en pro del desarrollo de la competencia mediática e informacional. En 2020, "la ubicuidad de la desinformación y el papel crucial del conocimiento de los agentes envueltos en la misma y del desarrollo del pensamiento crítico para afrontarla obligan a la actualización de este currículo" (Alcolea-Díaz, Reig y Mancinas, 2020) desde un enfoque estructural que favorezca una ciudadanía crítica.

Una corriente que busca dar respuesta a los desafíos que se deben afrontar y los obstáculos que se deben superar para adaptar la educación en medios al nuevo contexto crítico y participativo. Así lo ponen de relieve también Fedorov y Leviskaya (2015) al reconocer la necesidad de mejorar la educación en medios para lograr una ciudadanía crítica, contando con la participación de organizaciones sociales. La globalización proporciona oportunidades de cambio y de interconexiones, como reconocen Frau-Meigs y Torrent (2009). En este marco, Buckingham (2009) plantea algunos cambios en el plano político que requieren la implicación de los organismos internacionales y la sensibilización de la ciudadanía en el desarrollo de las políticas globales que fortalezcan la educación mediática de niños y jóvenes, de una ciudadanía mediática, en definitiva, tal y como lo plantea Gozálvez (2013).

Por ello, como recogen Frau-Meigs y Torrent (2009), organizaciones intergubernamentales como UNESCO, IESCO (Organización Islámica para la Educación, la Ciencia y la Cultura), la Liga de los Estados Árabes, SEGIB (Secretaría General Iberoamericana), la Alianza de Civilizaciones, la Comisión Europea, el Consejo de Europa o el Centro Internacional de Intercambio de Información sobre Ni- 
ños, Jóvenes y Medios de Comunicación de Nordicom, entre otros, apoyan desde hace tiempo la necesidad de un desarrollo sostenible, coherente y mundial que posicione a la alfabetización mediática como precursora de la inclusión digital.

Sin embargo, como alertan Ponte y Contreras-Pulido (2013: 19), "mientras que el mundo de los medios y de las tecnologías avanza a ritmos desorbitados, estas políticas y medidas lo hacen lentamente".

\section{LA ALFABETIZACIÓN MEDIÁTICA EN EL ENTORNO UNESCO, ONU Y UE}

Un avance significativo desde el punto de vista de las políticas en educación y comunicación es que las estrategias de esta última década ya tienen la vista puesta en el nuevo entorno convergente. UNESCO, por ejemplo, aplica también la convergencia en sus estrategias. Por un lado, en su estructura convergen las múltiples Cátedras Unesco de Comunicación en la nueva Red Orbicom, que, tras el encuentro fundacional de París en 2012, reunió en Marruecos en julio de 2013 experiencias de países de los cinco continentes. Desde entonces, y hasta el último encuentro de Estraburgo en 2019, esta red implementa sus debates globales poniendo el foco en retos tan importantes como la libertad de expresión en la era digital. Por otra parte, UNESCO trabaja desde hace varios años en la convergencia de los que tradicionalmente eran dos campos separados y diferentes: la alfabetización mediática, por un lado, y la alfabetización informacional, por otro. En 2011 se publica la conocida como AMI —estrategia sobre Alfabetización Mediática e Informacional (Wilson, Grizzle, Tuazon, Akyempong y Cheung, 2011)_, que con la UNESCO ofrece un recurso útil para alcanzar los objetivos propuestos en más de treinta años de declaraciones, desde la Declaración Grünwald (1982) hasta la Declaración de Riga (2016), durante la celebración del Foro Europeo de Alfabetización Mediática e Informacional, y pasando por la Declaración de Alejandría (2005) o la Agenda de París de la UNESCO (2007). Se plantean recomendaciones para reforzar la alfabetización mediática e informacional a los diferentes colectivos implicados (educadores y responsables del ámbito cultural y audiovisual, responsables de los medios, sociedad civil). El conocimiento de las funciones de algunos proveedores de información (medios, archivos, bibliotecas, etc.), así como de las condiciones en las que estas se desarrollan, permitirá que los usuarios se involucren en las dinámicas de los medios y apliquen un pensamiento para exigir a empresas de comunicación y otros proveedores de información un servicio de alta calidad.

El documento New Curriculum for Teachers on Media and Information Literacy forma parte de una amplia estrategia cuya finalidad es lograr que las sociedades sean alfabetizadas en medios para promover la cooperación internacional. Por eso, entre sus objetivos se incluye el desarrollo de acciones como la creación de un Centro de Intercambio de Información en colaboración con la Alianza de Civilizaciones de Naciones Unidas; el impulso de una red universitaria, la preparación de un marco global de los indicadores AMI o la articulación de lineamientos base para las políticas y estrategias nacionales. 
Precisamente la Alianza de las Civilizaciones, a través de la sección Media Literacy, impulsa la página web Educación en Medios (www.aocmedialiteracy. org) y la publicación del documento Mapping Media Education in the World, elaborado ya en un contexto en el que se detecta una pérdida de la dimensión humanística de la educación en medios, influenciada por un proceso de crisis económica con recortes que suponen una barrera en el acceso a las TIC (PérezTornero y Pi, 2014). Frente a esta situación, se evidencia, sin embargo, un aumento en el consumo de tecnologías y servicios, por lo que los ciudadanos necesitan dominar una serie de competencias mediáticas y digitales que les garantice un uso adecuado, responsable y seguro de los recursos y tecnologías.

El UNESCO MIL Curriculum and Competency Framework combina las áreas de alfabetización mediática y alfabetización informativa. La Unión Europea también marca como objetivo prioritario el logro de una alfabetización mediática en el entorno digital. La estrategia de apoyo internacional a la educación en medios a través del Programa Media de la Comisión Europea fructifica en una línea de atención específica: Media Literacy (<http://ec.europa.eu/culture/media/ media-literacy/index_en.htm>), vinculada a Europa Creativa 2014-2020. En este entorno se recogen las aportaciones del Grupo de Expertos que entre 2006 y 2010 trabajó en el diseño de la estrategia común europea en este ámbito, además de una mirada a la situación en el entorno mundial a través del Media Literacy Worldwide (Media 2007-2013).

Los antecedentes de la política europea se encuentran en la Recomendación 2009/625/CE de la Comisión Europea. Con el fin de establecer una estrategia común en el entorno europeo, la Comisión entiende que la alfabetización mediática en el contexto digital debe avanzar vinculada a proyectos de investigación interdisciplinares y transversales que permitan dar respuesta a los nuevos retos que ya se recogían en la Comunicación de 2007, titulada "Un planteamiento europeo de la alfabetización mediática en el entorno digital", y que luego fructificaría en la iniciativa i2010. La recomendación surgida entonces implicaba tres líneas de acción sobre los contenidos en línea, la comunicación comercial y las obras audiovisuales, y marcaban tres estrategias: implementar la participación en iniciativas de alfabetización mediática de autores nacionales y responsables de la regulación de medios audiovisuales y comunicaciones electrónicas; fijar la necesidad de determinar nuevos criterios de evaluación a través del seguimiento permanente de las iniciativas de alfabetización en medios; y elaborar códigos de conducta o marcos normativos que implicasen a distintos agentes interesados (autoridades nacionales, consumidores, medios de comunicación y otros productores y proveedores de contenidos, instituciones culturales y de investigación, así como centros de enseñanza). En 2018, el Parlamento Europeo (Comisión Europea, 2018) aprobó la Resolución sobre la educación en la era digital: retos, oportunidades y lecciones para el diseño de las políticas de la Unión (2028/2090(INI)) con base al Plan de Acción de Educación Digital y con cinco objetivos estratégicos, que se concretan en tres grandes prioridades:

1. Hacer un mejor uso de la tecnología digital para la enseñanza y el aprendizaje. 
2. Desarrollar competencias y habilidades digitales de relevancia para la transformación digital.

3. Mejorar los sistemas educativos a través del análisis de datos y procesos de previsión.

\section{ACTUALIZACIÓN DE LAS LÍNEAS ESTRATÉGICAS DE LA 'MEDIA LITERACY'}

En un contexto mediático dominado por el componente de participación social en los medios de comunicación, que explosiona con la convergencia tecnológica (especialmente en el entorno televisivo con la fusión de la TV con internet y las redes sociales, connected-TV/social-TV), se impone una realidad transmediática, es decir, un cruce de caminos (cross-media) convergente en un mismo punto: los intensos y acelerados cambios en los usos y consumos de los medios en torno a los que se generan unas expectativas de aprovechamiento en múltiples dimensiones. Capacitar a las audiencias, especialmente a los escolares, para que sean capaces de interpretar e imprimir sentido crítico a este consumo en los diferentes soportes de difusión de la comunicación, constituye el principal reto en torno al que se deben diseñar estrategias para la actual educación en comunicación y lograr una mayor participación en el ámbito comunitario a través de la conexión a internet (Buckingham, 2000, 2003; Barber, 2009; Hobbs, 2011). En la misma línea, Torrent (2016) desde la UNESCO reclama un mayor protagonismo a los medios de comunicación producidos por jóvenes, como actividad central de la educación mediática en los centros educativos, que proporcione nuevas plataformas para la inclusión social, en la línea de los Objetivos de las Naciones Unidas para el Desarrollo Sostenible.

Y una de esas líneas estratégicas pasa por avanzar en el desarrollo de la transliteracy que aglutina la capacidad de lectura, escritura e interacción a través de una variedad de plataformas, herramientas y medios como los impresos, la televisión, la radio, el cine o las redes sociales digitales. Este proceso de "alfabetización trans-" desde el año 2005 centra la línea de investigación del profesor Alan Liu, de la Universidad de California (EE UU) en la que convergen las humanidades, las ciencias sociales y la ingeniería. Posteriormente, el profesor Sue Tomas, de la Universidad de Montfort-Leicester (Reino Unido) crea el grupo PART añadiendo la producción al enfoque de la alfabetización en medios. Sin embargo, para FrauMeigs (2013), la transliteracy trata de fomentar un sistema más integrado en los contenidos culturales abriendo nuevas perspectivas en el aprendizaje a distancia, ya que las nuevas herramientas de comunicación facilitan el diálogo cultural con alumnos, que se reúnen en torno a un proyecto de aprendizaje que se refuerza con una presencialidad virtual (e- presence). La conectividad que permiten las redes sociales y de mensajería instantánea (Instagram, Snapchat, TikTok, Facebook, Twitter, Whatsapp, Telegram, etc.) y otras herramientas de conexión a distancia a través de la imagen y el sonido (Skype, Hangouts de Google, FaceTime de Apple, Microsoft Teams, etc.) permiten avanzar en la construcción de comunidades de intereses de aprendizaje, siguiendo el modelo que Jenkins (2008) define 
en sus "comunidades virtuales" en torno a las que también puede converger el aprendizaje mixto (blended learning). Por eso, reivindicar un espacio central para el desarrollo de la transliteracy en los currículos escolares, que permita su aplicación de forma transversal entre las diferentes áreas de conocimiento, ha de ser otra de las acciones estratégicas para avanzar en la educación en comunicación. Aguaded y Delgado-Ponce (2012) sostienen que, en el caso español, el papel de la educación mediática en el currículum no es suficiente ni comparable con la influencia o la presencia que tienen las TIC o los propios medios; si bien se están logrando hitos importantes al reconocerse expresamente, y por primera vez en la Ley 26/2015, de 28 de julio, de modificación del sistema de protección a la infancia y a la adolescencia, la importancia de la alfabetización digital y mediática como herramienta imprescindible para que los menores estén en disposición de desarrollar la competencia mediática, con el fin de poder formar parte activa de la sociedad. En clave española, la Agenda Digital publicada en febrero de 2013 podría ejercer de elemento corrector si se lleva a cabo uno de los seis grandes objetivos que plantea en el escenario de 2020: la alfabetización digital y la creación de nuevos perfiles profesionales en el ámbito TIC.

En este punto se refuerza otra línea estratégica que afecta a la transversalidad propia de la educación en medios, que es averiguar cómo afecta la crisis económica a la aplicación de las "macro-políticas" en media literacy recogidas, entre otras, en la estrategia MIL y sus esfuerzos por reforzar sus dos vertientes: la productiva y participativa y la analítica y crítica (Pérez-Tornero y Tayie, 2012). Áreas clave de un currículum que Tyner, Gutiérrez y Torrego (2015) complementan con una serie de recomendaciones para su implementación, como la importancia de permitir la interconexión entre el aprendizaje formal y el aprendizaje informal enriquecido por el uso y consumo de medios por niños y jóvenes. Sobre el papel decisivo de los docentes, González, Gozálvez y Ramírez (2015) aportan también una serie de recomendaciones para diseñar una propuesta de formación específica para integrar en el currículum escolar la educación mediática y digital. La alfabetización mediática y digital requiere la colaboración de todos los agentes educativos, acompañada de decisiones políticas que definan las líneas a seguir (Buckingham, 2009) para ayudar a niños y jóvenes a convertirse en consumidores y productores de mensajes de una manera responsable, ética y creativa, pero sin olvidar aspectos sustanciales como los que plantean Hobbs y Jensen (2009) respecto a la identidad individual y social, la interacción y la definición de límites entre lo público y lo privado, o las cuestiones legales y éticas vinculadas a los mensajes audiovisuales. Más aun teniendo en cuenta que la educación mediática se desarrolla a lo largo de toda la vida (Buckingham, 2000), es necesario preparar a los jóvenes, más que protegerles, para que se conviertan en ciudadanos libres y aprovechen los recursos en todos los ámbitos de su vida. Una línea de acción estratégica a escala mundial para avanzar en la alfabetización digital es fomentar la investigación. Desde 2013, la alfabetización mediática refuerza los estudios europeos sobre medios. Además de las líneas ya expuestas en torno al programa Media Literacy, y como resultado de la conferencia "Estudios de medios: Nuevos medios y nuevas alfabetizaciones", que reunió en Eslovenia a investigadores, responsables políticos y profesionales, la Fundación Europa de la Ciencia colocó 
encima de la mesa una agenda estratégica común para desplegar el compromiso de una política científica acorde a la importancia de integrar estas cuestiones y realizar cambios en la esfera política, donde siguen plenamente vigentes las razones para la Educación en Medios que posicionaba Aguaded (2001).

\section{DISCUSIÓN Y CONCLUSIONES}

A pesar de todo lo avanzado en estos años de intenso trabajo, los interrogantes que surgen ante los nuevos desafíos son muchos y muy importantes, tal y como se ha recogido a partir de las categorías de análisis de los documentos consultados planteado en el primer objetivo del estudio. La complejidad de las nuevas relaciones entre medios y usuarios en el entorno digital, dominado por la convergencia, hace saltar las alarmas ante los peligros y riesgos de no tener la preparación adecuada para ejercer un uso correcto de las nuevas herramientas de comunicación. Sin embargo, también abre un sinfín de oportunidades que, con un correcto aprovechamiento, pueden ser de gran utilidad. En esta línea, el informe Forward Look Media in Europe: New Questions for Research and Policy de la European Science Foundation (2014) posiciona en sus conclusiones algunas líneas estratégicas para avanzar en las reflexiones y propuestas. Una de ellas deja explícitamente abierta la puerta a investigar cómo la alfabetización mediática (media literacy) debe servir para fomentar el valor de la ciudadanía y mejorar la participación, el empoderamiento social y la inclusión cultural. "La alfabetización audiovisual en sus diversas construcciones", dice este informe, "debe ayudar a que los consumidores sean capaces de entender los procesos mediante los cuales los medios ofrecen comunicación y admiten su participación como productores, reforzando su perspectiva de consumo y aportación crítica". Del mismo modo, según la propuesta de Pérez-Escoda, García-Ruiz y Aguaded (2019), la alfabetización digital debe formar parte de la educación mediática de los ciudadanos, y favorecer así el desarrollo de las áreas competenciales que garantizarán su correcto desempeño en la convergencia mediática actual.

Teniendo en cuenta que uno de los factores determinantes de la educación en los medios son las capacidades económicas, queda por analizar los efectos de la crisis económica a escala mundial en la formación del profesorado en alfabetización informativa y mediática, que se declaró como "reto candente en el sistema educativo mundial" (Pérez-Tornero y Tayie, 2012) y que da respuesta al segundo de los objetivos planteados en este estudio. En este sentido, los recortes de los recursos pueden estar condicionando el presente y el futuro de todo un conjunto de cuestiones que forman parte del debate a corto y medio plazo, y que podrían agruparse en torno a estos interrogantes:

1. Qué: necesidad de seguir avanzando en redefinir el concepto y adaptar la educación en medios al actual escenario. La digitalización y la consiguiente convergencia mediática han cambiado las formas de actuar, de comunicarse y de relacionarse, de acceder al conocimiento y de consumir entretenimiento e información. Las discusiones en torno a las nuevas tecnologías y la 
alfabetización digital pasan por integrar el acceso y la interpretación de los contenidos en los nuevos continentes o soportes, lo que supone estar alfabetizado (Potter, 2010). La nueva educación en medios debe profundizar en el conocimiento crítico que exige el nuevo entorno a las audiencias activas. El desafío está en seguir buscando el espacio en los discursos de las nuevas alfabetizaciones digitales desde una postura crítica de las representaciones mediáticas. En el III Congreso Internacional de Educación Mediática y Competencia Digital (\#Edumed17), que recogió el eje Media Education Summit 2017 promovido por el Centre for Excellence in Media Practice, los expertos posicionaron con insistencia la necesidad de profundizar en la materia de educación y comunicación pensando en integrar nuevos elementos en las políticas de educación mediática como la gamificación o la inteligencia emocional (Gutiérrez, García-Matilla y Collado, 2017). La UNESCO reforzó el ámbito de las redes sociales con su estrategia MIL CLICKS (<https:// en.unesco.org/MILCLICKS>) hacia la adquisición de competencias para la alfabetización mediática e informacional (AMI) en el ámbito del acceso cotidiano a internet y las redes sociales que realizan especialmente los jóvenes a través de las búsquedas, los juegos, conectándose, compartiendo y socializando. La propuesta es clara: revisar los viejos y nuevos alfabetismos y apostar por profundizar en el aprendizaje mediático.

2. Quién: o mejor, quiénes, deben ser los agentes que sostengan el esfuerzo que se debe hacer en el nuevo contexto. Las intenciones de las políticas públicas propuestas por los órganos reguladores deben contar con el respaldo de los stakeholders de la educación: desde las familias hasta las escuelas, incluyendo otras instituciones educativas, ONG-asociaciones y, por supuesto, los medios de comunicación.

3. Cómo: hay muchos interrogantes que necesitan despejarse a corto y medio plazo. Quizás, el principal, del que dependen los demás, sería cómo afrontar la nueva alfabetización en convergencia, donde la tradicional separación entre soportes se integra en una nueva dimensión. Se debe avanzar en la aplicación de lo que Jenkins (2008) posiciona como una nueva cultura de la participación derivada de la inteligencia colectiva y colaborativa. Su explosión se produce en un contexto de convivencia e integración de los viejos con los nuevos medios, y en donde la comunicación alcanza nuevas cotas de bidireccionalidad al confundirse e intercambiarse los roles entre productores/creadores de productos comunicativos y consumidores/usuarios de los mismos. Una segunda incógnita pasa por, ¿cómo manejar el peligro que puede suponer la fascinación por el potencial de la tecnología en la educación mediática 2.0 y 3.0, en detrimento del espíritu crítico o la reflexión inspirados en pedagogías como la de Paulo Freire? Y para dar respuesta a todo ello, ¿cómo seguir fomentando la integración en los currículums escolares de la educación en medios?

4. Dónde: en la globalidad que debe respetar la diversidad. Es la convergencia de las distintas áreas en una política global capaz de unificar los criterios que deben marcar las nuevas estrategias y de diseñar un currículum íntegro, que dé respuesta a diferentes realidades, desde lo global hasta lo local, primando 
la inclusión y siendo conscientes de los límites de los recursos tecnológicos (Gozálvez, 2013).

5. Cuándo: en el presente más inmediato y en un futuro próximo. Las tecnologías avanzan a un ritmo veloz y lo que hoy empezamos a interpretar puede quedarse obsoleto en muy poco tiempo. Es necesaria una rápida capacidad de reacción para la acción.

6. Por qué: porque asumir los riesgos de un avance equivocado del conocimiento tecnológico en las distintas etapas del proceso de aprendizaje aumenta los peligros y las consecuencias negativas que se pueden derivar de estos errores.

Este trabajo se ha desarrollado al amparo del Proyecto español I+D+I "Youtubers e instagrammers: La competencia mediática en los prosumidores emergentes" (RTI2018-093303-B-I00) (MINECO/FEDER).

Ana Isabel Rodríguez-Vázquez (anaisabel. rodriguez.vazquez@usc.es). Docente en la Universidad de Santiago de Compostela. Doctora en Comunicación por la USC y licenciada en Ciencias de la Información por la Universidad Complutense de Madrid. Trabajó en prensa, televisión e internet. Imparte materias sobre géneros informativos, programación y audien-

Rosa García-Ruiz (rosa.garcia@unican.es). Profesora de la Universidad de Cantabria, del área de Didáctica y Organización Escolar y colaboradora del Doctorado Interuniversitario en Comunicación (UHU) y de la Universidad Nebrija. Editora adjunta de la revista Comunicar y evaluadora de más de quince revistas. Ha publicado artículos sobre educación mediática y metodología docente en revistas JCR y SCOPUS, libros, capítulos de libro y numerosas aportaciones en congresos internacionales sobre educación y alfabetización mediática.

Ignacio Aguaded (aguaded@uhu.es). Catedrático de Universidad de Educación y Comunicación en la Universidad de Huelva. Preside el Grupo Comunicar. Es editor de la revista científica de comunicación y educación $\mathrm{Co}$ - cias en los grados de Periodismo y Comunicación Audiovisual, y en el Máster en Periodismo y Comunicación. Pertenece al Grupo de Investigación Estudios Audiovisuales (GEA) de la USC, es miembro de la red Real Code y colabora en proyectos de grupos como Novos Medios (USC) o Fonta (UCM) a través de proyectos competitivos estatales e internacionales.

Miembro de comités científicos y organizadores de diferentes congresos internacionales. Ha realizado estancias en universidades nacionales, en Estados Unidos, Perú e Italia. Miembro de Alfamed (Red Interuniversitaria Euroamericana de Investigación en Competencias Mediáticas para la Ciudadanía) y de la Red de Excelencia en Educación Mediática (EDUMED). Investigadora en Proyectos competitivos de ámbito nacional (Ministerio de Ciencia, Innovación y Universidades) y europeo (H2020, Erasmus+).

municar, investigador principal del Grupo de Investigación Ágora dentro del Plan Andaluz de Investigación (HUM-648) de la Universidad de Huelva, presidente de Alfamed (Red Interuniversitaria Euroamericana de Investigación 
en Competencias Mediáticas para la Ciudadanía) y responsable de la Red de Excelencia en Educación Mediática (EDUMED). Premio de Mejor Investigador de la Universidad de Huelva 2105. Ha participado en múltiples actividades formativas y de investigación en el ámbito del uso didáctico de los medios de comunicación y ha dictado conferencias en foros y congresos de universidades nacionales y extranjeras, $y$ ha participado con ponencias en másteres de diferentes universidades españolas, así como en centros de investigación extranjeros. Director del Programa Doctorado Interuniversitario en Comunicación, de la línea de investigación Educomunicación. Director del Máster Interuniversitario en Comunicación y Educación Audiovisual (UHU y UNIA). Investigador principal de numerosos proyectos de investigación competitivos, nacionales e internacionales.

\section{Bibliografía}

Aguaded, I. (2001). La educación en medios de comunicación. Panorama y perspectivas. Murcia: KR.

Aguaded, I. y Delgado-Ponce, Á. (2012). "La media education in Spagna". Media Education, 3. Disponible en: <http://riviste.erickson. it/med/it/2012/la-media-education-in-spagna/>. Consultado el 10 octubre de 2019.

Alcolea-Díaz, G.; Reig, R. y Mancinas, R. (2020). "Currículo de Alfabetización Mediática e Informacional de la UNESCO para profesores desde una perspectiva de la Estructura de la Información". Comunicar, 62, pp. 103-114. <https://doi.org/10.3916/C62-2020-09> Consultado el 8 de marzo de 2020.

Álvarez-Arregui, E. y Rodríguez-Martín, A. (2015). "Inspirando el cambio en educación: Ecosistemas de formación para aprender a emprender". Estudios pedagógicos, 41 (especial), pp. 9-29. Disponible en: <https://doi. org/10.4067/S0718-07052015000300002> Consultado el 1 de junio de 2019.

Álvarez-Arregui, E.; Rodríguez-Martín, A.; Madrigal-Maldonado, R.; Grossi-Sampedro, B. y Arreguit, X. (2017). "Ecosystems of Media Training and Competence: International Assessment of Its Implementation in Higher Education" ["Ecosistemas de formación y competencia mediática: Valoración internacional sobre su implementación en la educa- ción superior"]. Comunicar, 51, pp. 105-114. <https://doi.org/10.3916/C51-2017-10> Consultado el 5 de septiembre de 2019.

Andersen, N. (2016). "Curriculum Contexts. How Might Context Influence or Inflect Media Literacy Education?" The Journal of Media Literacy, 63 (1-2), pp. 60-69.

Barber, T. (2009). "Participation, Citizenship, and Well-being En-gaging with Young People, Making a Difference". Young, 17 (1), pp. 25- 40. Disponible en: <https://doi.org/10 $.1177 / 110330880801700103>$. Consultado el 12 de julio de 2019

Buckingham, D. (2000). "Infancias cambiantes, medios cambiantes: nuevos desafíos para la educación mediática". Cultura y Educación, 20, pp. 23-38.

- (2003). "Media Education and the End of the Critical Consumer". Harward Educational Review, 73 (3), pp. 309-327.

-. (2009). "The Future of Media Literacy in the Digital Age: some Challenges for Policy and Practice". Medienimpulse, 2, pp. 69-82.

Castro, A.; Renés, P. y Phillippi, A. (2016). "Media Literacy in the School Curriculum". The Journal of Media Literacy, 63 (1-2), pp. 18-27.

Comisión Europea (2018). "Comunicación de la Comisión al Parlamento Europeo, al Consejo, al Comité Económico y Social Europeo y al Comité de las Regiones sobre el Plan 
de Acción de Educación Digital". Disponible en: <https://eur-lex.europa.eu/legal-content/ $\mathrm{ES} / \mathrm{TXT} / \mathrm{PDF} /$ ?uri=CELEX:52018DC0022\&fr om=EN> Consultado el 12 de diciembre de 2019.

Dezuanni, M. (2016). "National Educational Policy for Media Literacy Education: Media Arts in the Australian Curriculum". The Journal of Media Literacy, 63 (1-2), pp. 28-33.

Fedorov, A. y Levitskaya, A. (2015). "Situación de la educación en medios y la competencia crítica en el mundo actual: opinión de expertos internacionales". Comunicar, 45, pp. 107-116. <https://doi.org/10.3916/C452015-11>.

Frau-Meigs, D. (2013). "Future Trends in the Matter of Media and Information Literacy". Comunicación presentada en el II Congreso Internacional Educación mediática \& competencia digital. Ludoliteracy, creación colectiva y aprendizajes, Barcelona. Disponible en: <www.uoc.edu/portal/es/symposia/congreso_ludoliteracy2013/conclusiones_actas/ actas_definitivas_congreso_edumed_2013. pdf $>$. Consultado el 15 de enero de 2019.

Frau-Meigs, D. y Torrent, J. (2009). "Políticas de educación en medios. Hacia una propuesta global". Comunicar, 32, pp. 10-14.

Freire, P. (1967). Educação como prática da liberdade. Río de Janeiro: Paz e Terra. Disponible en: <www.dhnet.org.br/direitos/militantes/paulofreire/livro_freire_educacao_pratica_liberdade.pdf $>$. Consultado el 10 de enero de 2019.

García-Ruiz, R.; Matos, A. y Borges, G. (2016). "Media Literacy As a Responsability of Families and Teachers". The Journal of Media Literacy, 63 (1-2), pp. 82-91.

Goble, R. (2016). "Reexaminig the Early History of American Media Education \& Literacy". The Journal of Media Literacy, 63 (1-2), pp. 70-81.

González, N.; Gozálvez, V. y Ramírez, A. (2015). "La competencia mediática en el profesorado no uniRecuperado de:sitario. Diagnóstico y propuestas formativas". Revista de
Educación, 367, pp. 117-146. <hpps://doi. org/10.4438/1988-592X-RE- 2015-367-285>.

Gozálvez, V. (2013). Ciudadanía mediática. Una mirada educativa. Madrid: Dykinson

Gutiérrez-Martín, A. (2013). "Educación mediática en la era de la convergencia". Comunicación presentada en el II Congreso Internacional de Educación Mediática y Competencia Digital. Recuperado de <http:// www.uoc.edu/portal/es/symposia/congreso_ ludoliteracy2013/programa/ACTAS_EDUMED _2013.pdf>.

Gutiérrez, A.; García-Matilla, A. y Collado, R. (eds.) (2017). "Educación mediática y competencia digital". Comunicación presentada en el III Congreso Internacional de Educación Mediática y Competencia Digital. Disponible en: <http://www.educacionmediatica.es/ inscripcion-y-comunicaciones/>. Consultado el 3 de junio de 2019.

Gutiérrez, J. J. y Gómez, M. T. (2015). “Influencia de las TIC en los procesos de aprendizaje y comunicación de los estudiantes de educación". Revista de Pedagogía, 35 y 36, (9798), pp. 34-51.

Hobbs, R. (2011). Digital and Media Literacy: Connecting Culture and Classroom. California: Corwin.

Hobbs, R.; Donnely, K.; Friesem. J. y Moen, M. (2013). Learning to Engage: How Positive Attitudes about the News, Media Literacy, and Video Production Contribute to Adolescent Civic Engagement. Educational Media International, 50 (4), pp. 231-246. Recuperado de <http://www.tandfonline.com/doi/abs/10.1 080/09523987.2013.862364\#.VHyR9xajTfN> Consultado el 10 de enero de 2019.

Hobbs, R. y Jensen, A. (2009). "The Past, Present, and Future of Media Literacy Education". Journal of Media Literacy Education 1, pp. 1-11. Recuperado de $<\mathrm{http}$ //digitalcommons. uri.edu/jmle/vol1/iss1/1/>. Consultado el 10 de enero de 2019.

Jenkins, H. (2008). Convergence Culture: La cultura de la convergencia de los medios de comunicación. Madrid: Paidós. 
Lee, A. Y. L. (2016). "Learning Media Literacy in Urban and Rural Schools in China". The Journal of Media Literacy, 63 (1-2), pp. 3442.

Martens, H. y Hobbs, R. (2015). "How Media Literacy Supports Civic Engagement in a Digital Age". Atlantix Journal of Communication, 23, pp. 120-137. <https://doi.org/10.108 0/15456870.2014.961636>.

Pérez-Escoda, A.; García-Ruiz, R. y Aguaded, I. (2019). "Dimensions of Digital Literacy Based on Five Models of Development". Cultura y Educación, 31, pp. 232-266. <https:// doi. org/10.1080/11356405.2019.1603274>.

Pérez-Tornero, J. M. y Tayie, S. (2012). "La formación de profesores en educación en medios: Currículo y experiencias internacionales". Comunicar, 39, pp. 10-14. <https://doi. org/10.3916/C39-2012-02-00>.

Pérez-Tornero, J. M. y Pi, M. (2014). "La educación en medios en una España en crisis". En: Elea, I. (ed.). Agentes e Voces. Um panorama da Mídia-Educação no Brasil, Portugal e Espanha, pp. 247-256. Estocolmo: Nordicom.

Pérez-Tornero, J. M. (2015). "La emergencia de la alfabetización mediática e informacional Un nuevo paradigma para las políticas públicas y la investigación". Telos, 100, pp. 99102. Disponible en: <https://telos.fundaciontelefonica.com/url-direct/pdf-generator?tipoC ontenido $=$ articuloTelos\&idContenido $=20150$ $32317440002 \&$ idio ma=es $>$. Consultado el 10 de enero de 2019.

Ponte, C. y Contreras-Pulido, P. (2013). "Presente y futuro de la alfabetización mediática en Europa: el caso español y portugués". Chasqui, 124, pp. 19-25.

Potter, W. J. (2010). "The State of Media Literacy". Journal of Broadcasting \& Electronic Media, 54 (4), pp. 675-696.
Torrent, J. (2016). "Youth-Produced Media: Platforms for Social Inclusion". The Journal of Media Literacy, 63 (1-2), pp. 110-113.

Tyner, K. (2013). "Blended Learning: Connecting Media Analysis and Practice". Comunicación presentada en el II Congreso Internacional Educación mediática y competencia digital. Ludoliteracy, creación colectiva y aprendizajes, Barcelona. Disponible en www.uoc.edu/portal/es/symposia/congreso_ludoliteracy2013/conclusiones_actas/ actas_definitivas_congreso_edumed_2013.pdf. Consultado el 10 de enero de 2019.

Tyner, K.; Gutiérrez, A. y Torrego, A. (2015). "Multialfabetización sin muros en la era de la convergencia. La competencia digital y "la cultura del hacer" como revulsivos para una educación continua". Profesorado. Revista de Curriculum y Formación del Profesorado, 19 (2), pp. 41-56.

European Science Foundation (2014). Forward Look Media in Europe: New Questions for Research and Policy. Disponible en: $<$ www.esf.org/fileadmin/Public_documents/ Publications/media_in_europe_01.pdf $>$. Consultado el 8 de enero de 2019.

Valdivia-Barrios, A.; Herrera, M. y Guerrero, M. (2015). “Aprendizaje y producción mediática digital en la escuela: Un abordaje etnográfico del aprendizaje como práctica cultural en Artes Visuales". Estudios Pedagógicos, 41 (especial), pp. 231-251. <https://doi. org/10.4067/S0718-07052015000300015>.

Wilson, C.; Grizzle, A.; Tuazon, R.; Akyempong, K. y Cheung, Ch. K. (2011). Alfabetización mediática e informacional. Curriculum para profesores. Nueva York.: UNESCO. Disponible en: <http://unesdoc.unesco.org/ images/0021/002160/216099S.pdf>. Consultado el 11 de abril de 2019. 
